\title{
Self-similar and disordered front propagation in a radial Hele-Shaw channel with time-varying cell depth
}

\author{
C. Vaquero-Stainer, ${ }^{1}$ M. Heil, ${ }^{2}$ A. Juel, ${ }^{1}$ and D. Pihler-Puzović ${ }^{1, *}$ \\ ${ }^{1}$ Manchester Centre for Nonlinear Dynamics and School of Physics and Astronomy, University of Manchester, \\ Oxford Road, Manchester M13 9PL, United Kingdom \\ ${ }^{2}$ Manchester Centre for Nonlinear Dynamics and School of Mathematics, University of Manchester, \\ Oxford Road, Manchester M13 9PL, United Kingdom
}

(Received 3 March 2019; published 6 June 2019)

\begin{abstract}
The displacement of a viscous fluid by an air bubble in the narrow gap between two parallel plates can readily drive complex interfacial pattern formation known as viscous fingering. We focus on a modified system suggested recently by Zheng et al. [Phys. Rev. Lett. 115, 174501 (2015)], in which the onset of the fingering instability is delayed by introducing a time-dependent (power-law) plate separation. We perform a complete linear stability analysis of a depth-averaged theoretical model to show that the plate separation delays the onset of nonaxisymmetric instabilities, in qualitative agreement with the predictions obtained from a simplified analysis by Zheng et al. We then employ direct numerical simulations to show that in the parameter regime where the axisymmetrically expanding air bubble is unstable to nonaxisymmetric perturbations, the interface can evolve in a self-similar fashion such that the interface shape at a given time is simply a rescaled version of the shape at an earlier time. These novel, self-similar solutions are linearly stable but they only develop if the initially circular interface is subjected to unimodal perturbations. Conversely, the application of nonunimodal perturbations (e.g., via the superposition of multiple linearly unstable modes) leads to the development of complex, constantly evolving finger patterns similar to those that are typically observed in constant-width Hele-Shaw cells.
\end{abstract}

DOI: 10.1103/PhysRevFluids.4.064002

\section{INTRODUCTION}

An expanding air bubble that displaces a viscous fluid within the narrow gap between two parallel plates (a Hele-Shaw cell) is unstable to nonaxisymmetric perturbations beyond a critical value of the capillary number $\mathrm{Ca}$, the ratio of viscous to surface tension forces [1-4]. In this radial geometry, the unstable interface typically deforms into a set of growing fingers which evolve continuously in time through a sequence of tip-splitting events followed by competition between the newly formed fingers. This nonlinear evolution is an archetype for front-propagating, pattern forming phenomena [5].

Longstanding interest in viscous fingering stems from its similarities with a range of other front propagation phenomena, such as the growth of bacterial colonies [6] and the solidification instabilities during crystal growth [7]. In hydraulic fracture used for oil recovery, viscous fingering is promoted to ensure heterogeneous placement of particles (proppants) into fractures, thereby increasing their hydraulic conductivity, but it is also actively suppressed to avoid early breakthrough of water into adjacent production wellbores [8,9]. This has stimulated a recent resurgence in research effort to delay the onset of viscous fingering, e.g., by manipulating the geometry of the cell, either

\footnotetext{
*Corresponding author: draga.pihler-puzovic@manchester.ac.uk
} 
actively [10,11], or passively by using compliant cells [12-14]; by controlling the injection rate [15]; or by tuning the viscosity ratio of miscible fluid pairs [16].

In this paper, we consider fingering in a radial Hele-Shaw cell in which the width of the gap between the parallel bounding plates increases as a function of time. This effect is stabilising since it reduces the rate at which the axisymmetric bubble expands; in fact, if done sufficiently rapidly, the plate separation can bring the expansion of the bubble to a halt or even cause the bubble to contract. In a recent study, Zheng et al. [17] employed a simplified analysis, based on Paterson's classical results for the system with constant gap width [2], to examine the case when the gap width increases according to the power law $b^{*}\left(t^{*}\right)=b_{1}^{*} t^{* 1 / 7}$, where $b^{*}$ is the distance between the plates and $t^{*}$ is time. They not only confirmed that viscous fingering can be suppressed if the plates are separated sufficiently rapidly (i.e., for sufficiently large values of the constant $b_{1}^{*}$ ) but also predicted that the wave number $k_{\max }$ of the most rapidly growing small-amplitude perturbation to the axisymmetric bubble is independent of the bubble's radius. Furthermore, they performed experiments which showed that in the parameter range for which the axisymmetric bubble is unstable, a small number of finite-amplitude nonsplitting fingers tended to develop and that the number of these fingers was remarkably close to $k_{\max }$.

The observation of nonsplitting fingers is unusual because it contrasts with the typical sequence of tip-splitting and finger competition observed in experiments without plate lifting [3,4]. The findings of Zheng et al. [17] suggest that, following some initial transients, the finger growth may become self-similar in the sense that the finger shape at a given time is simply a rescaled version of the shape at an earlier time. This is analogous to proportionate growth observed in biological systems, e.g., during growth of a mammal whose body parts grow at nearly the same rate and thus in direct proportion to each other [18]. In radial Hele-Shaw cells without lifting, proportionate growth of fingering (or self-similar fingering) has been observed in some experiments with miscible fluids [16]. Self-similar growth of the interface between two immiscible fluids has previously been observed [19,20] and predicted [21-23] in a related setup where the cell consists of a disk sector. This geometry promotes the formation of a single finger which is symmetric about the sector centreline and which does not split for sufficiently small values of $\mathrm{Ca}$ or sector angles. Other, more complex but well-defined, reproducible finger shapes were also obtained by imposing selected initial perturbations. Moreover, Li et al. [24] have observed self-similarly evolving fingering patterns in a radial geometry with a fixed gap width by varying the air injection rate according to the power law $Q\left(t^{*}\right)=Q_{1} t^{*-1 / 3}$, where $Q$ is the injection flux. The number of finite-amplitude, self-similar fingers observed in their simulations matched the most unstable wave number obtained from a linear stability analysis. Intriguingly, these self-similar fingers were obtained in simulations which were started from an initial perturbation whose Fourier decomposition did not include the most unstable mode from the linear stability analysis. This indicates that in that system the self-similar fingers are formed through the nonlinear interaction of modes.

In this paper we revisit the scenario studied by Zheng et al. [17] and perform a full linear stability analysis to confirm that $k_{\max }$ is indeed independent of the bubble radius. We then perform numerical simulations of the system's nonlinear evolution following the onset of the linear instability. We demonstrate that self-similarly evolving fingering patterns can be realized with the lifting law introduced by Zheng et al. [17], but only when the initially circular interface is perturbed with a single, linearly unstable mode. Unimodal perturbations with wave number $k_{\max }$ lead to the development of $k_{\max }$ distinct nonsplitting, self-similar finite-amplitude fingers. If the interface is subjected to unimodal perturbations with other wave numbers the perturbed interface typically undergoes a transient phase during which fingers split before they approach a self-similar regime. Therefore, the number of self-similar fingers that emerge from the instability does not necessarily coincide with the most unstable wave number predicted by the linear stability analysis. We also find that if nonunimodal, random initial perturbations are introduced, such as those occurring in a typical experiment, the interface does not evolve towards a self-similar solution, in contrast with the scenario suggested by the experiments of Zheng et al. [17]. Instead, the fingering pattern evolves continuously as the interface advances across the cell through a succession of tip-splitting 


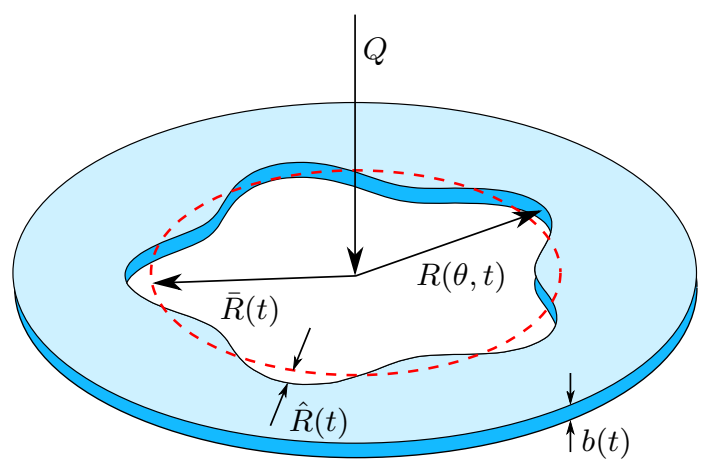

FIG. 1. Schematic diagram of a perturbed interface in the radial Hele-Shaw cell with time-varying gap width.

and finger competition events reminiscent of the patterns in a radial cell with fixed parallel plates.

\section{MATHEMATICAL MODEL}

We describe the motion of the wetting viscous fluid occupying the narrow gap between the two bounding plates using the lubrication approximation. Using horizontal polar coordinates $\left(r^{*}, \theta\right)$, centered at the injection point (see Fig. 1), the equations for the vertically averaged velocity $\boldsymbol{u}^{*}\left(r^{*}, \theta, t^{*}\right)$ and the fluid pressure $p^{*}$ are then

$$
\boldsymbol{u}^{*}=-\frac{b^{* 2}}{12 \mu} \nabla p^{*}, \quad \frac{d b^{*}}{d t^{*}}=-\nabla \cdot\left(b^{*} \boldsymbol{u}^{*}\right)=\frac{b^{* 3}}{12 \mu} \nabla^{2} p^{*} \quad \text { in } r^{*}>R^{*}\left(\theta, t^{*}\right),
$$

where $\mu$ is the liquid viscosity, $R^{*}\left(\theta, t^{*}\right)$ is the bubble radius, and $b^{*}\left(t^{*}\right)$ is the (spatially constant) gap width. Throughout this paper we employ asterisks to distinguish dimensional quantities from their nondimensional equivalents. In the region $r^{*}<R^{*}\left(\theta, t^{*}\right)$, the gas bubble has a uniform pressure $p_{g}^{*}\left(t^{*}\right)$. We neglect the effects of the thin films of liquid that are left behind the advancing bubble tip, and ignore viscous normal stresses at the interface. The kinematic and dynamic boundary conditions at $r^{*}=R^{*}\left(\theta, t^{*}\right)$ then become

$$
\begin{aligned}
\frac{\partial \boldsymbol{R}^{*}}{\partial t^{*}} \cdot \boldsymbol{n} & =\boldsymbol{u}^{*} \cdot \boldsymbol{n}, \\
p^{*}\left(R^{*}, \theta, t^{*}\right) & =p_{g}^{*}\left(t^{*}\right)-\gamma\left(\kappa_{\perp}^{*}+\kappa_{\|}^{*}\right)=p_{g}^{*}\left(t^{*}\right)-\gamma\left\{\frac{R^{* 2}+2\left(\frac{\partial R^{*}}{\partial \theta}\right)^{2}-R^{*} \frac{\partial^{2} R^{*}}{\partial \theta^{2}}}{\left[R^{* 2}+\left(\frac{\partial R^{*}}{\partial \theta}\right)^{2}\right]^{\frac{3}{2}}}+\frac{2}{b^{*}}\right\},
\end{aligned}
$$

where $\boldsymbol{n}$ is the unit normal to the interface and $\gamma$ is the surface tension. The mean curvature of the interface is approximated as the sum of the in-plane and transverse curvatures, $\kappa_{\|}^{*}$ and $\kappa_{\perp}^{*}$, respectively. At the outer boundary of the Hele-Shaw cell we impose $p^{*}\left(R_{\text {outer }}^{*}, \theta, t^{*}\right)=0$ (implying that all pressures are measured relative to the atmospheric pressure). Finally, for a constant injection flux $Q$, the volume of gas in the bubble is given by

$$
Q t^{*}=\frac{b^{*}\left(t^{*}\right)}{2} \int_{0}^{2 \pi} R^{* 2}\left(\theta, t^{*}\right) d \theta .
$$

We study the system's evolution starting from $t^{*}=t_{0}^{*}$ when the cell walls are separated by $b_{0}^{*}$ and the bubble has an initial radius $R^{*}=R_{\text {init }}^{*}$. 
In the following analysis, we nondimensionalize in-plane lengths with $R_{\text {outer }}^{*}$, the gap width with $b_{0}^{*}$, time with $2 \pi R_{\text {outer }}^{* 2} b_{0}^{*} / Q$ and pressures with $6 \mu Q / \pi b_{0}^{* 3}$. Then the problem is governed by three nondimensional parameters: the capillary number $\mathrm{Ca}=\mu Q /\left(2 \pi \gamma R_{\text {outer }}^{*} b_{0}^{*}\right)$, the cell aspect ratio $\mathcal{A}=b_{0}^{*} / R_{\text {outer }}^{*}$, and the initial radius of the bubble $R_{\text {init }}=R_{\text {init }}^{*} / R_{\text {outer }}^{*}$. At the initial time, when $t=t_{0}=R_{\text {init }}^{2} / 2$, we then have $b\left(t_{0}\right)=1$. When imposing Zheng et al.'s [17] power-law behavior for the plate separation, we have $b(t)=b_{1} t^{1 / 7}$, where $b_{1}=b_{1}^{*}\left[2 \pi R_{\text {outer }}^{* 2} /\left(b_{0}^{* 6} Q\right)\right]^{1 / 7}=t_{0}^{-1 / 7}=$ $\left(R_{\text {init }}^{2} / 2\right)^{-1 / 7}$.

\section{AXISYMMETRIC SOLUTIONS AND LINEAR STABILITY ANALYSIS}

Equations (1)-(4) have an axisymmetric solution for which the bubble radius is given by

$$
\bar{R}(t)=\left[\frac{2 t}{b(t)}\right]^{1 / 2},
$$

while the pressures in the viscous fluid and the gas bubble are

$$
\bar{p}(r, t)=\frac{1}{b^{3}} \frac{d b}{d t} \frac{r^{2}-1}{4}-\frac{1}{b^{3}} \log r
$$

and

$$
p_{g}(t)=\frac{1}{b^{3}} \frac{d b}{d t} \frac{\bar{R}^{2}-1}{4}-\frac{1}{b^{3}} \log \bar{R}+\frac{\mathcal{A}}{12 \mathrm{Ca}}\left(\frac{\mathcal{A}}{\bar{R}}+\frac{2}{b}\right)
$$

respectively. To assess the stability of this solution to nonaxisymmetric perturbations that change the shape of the interface, we assume $R(\theta, t)=\bar{R}(t)+\varepsilon \hat{R}_{k}(t) \sin (k \theta)$, where $\varepsilon \ll 1$ is the amplitude and $k>1$ is the integer wave number of the perturbation. A straightforward linear stability analysis then shows that the instantaneous growth rate $\lambda_{k}$ of the small-amplitude perturbation with wave number $k$ is given by

$$
\lambda_{k}=\frac{1}{\hat{R}_{k}} \frac{d \hat{R}_{k}}{d t}=\frac{1+\bar{R}^{2 k}}{1-\bar{R}^{2 k}} k\left[\frac{\mathcal{A}^{2} b^{2}\left(1-k^{2}\right)}{12 \mathrm{Ca} \bar{R}^{3}}-\frac{1}{b}\left(\frac{1}{2} \frac{d b}{d t}-\frac{1}{\bar{R}^{2}}\right)\right]-\frac{1}{b}\left(\frac{1}{2} \frac{d b}{d t}+\frac{1}{\bar{R}^{2}}\right) .
$$

For bubbles that are much smaller than the outer radius of the cell, we have $\left(1+\bar{R}^{2 k}\right) /\left(1-\bar{R}^{2 k}\right) \approx$ 1 , allowing us to approximate the growth-rate as

$$
\lambda_{k}=\frac{k-1}{\bar{R}^{2}}\left(\frac{1}{b}-\frac{\mathcal{A}^{2} b^{2} k(k+1)}{12 \mathrm{Ca} \bar{R}}-\frac{\bar{R}^{2}}{2 b} \frac{d b}{d t} \frac{k+1}{k-1}\right) .
$$

In the absence of lifting, i.e., $b=1$ and $d b / d t=0$, this reduces to Paterson's classical expression for the growth rate in an infinitely large Hele-Shaw cell with constant gap width [2]. A positive rate of plate separation, $d b / d t>0$, can be seen to reduce the growth rate of the perturbations and therefore stabilises the axisymmetric state.

We note that, in general, the instantaneous growth rate $\lambda_{k}$ of the small-amplitude perturbations varies with the evolving mean radius of the bubble. As a result, the range of unstable wave numbers (i.e., wave numbers for which $\lambda_{k}>0$ ) and the most unstable wave number (for which $d \lambda_{k} / d k=0$ ) generally change throughout the system's evolution. One of the key observations made by Zheng et al. [17] is that if the gap width has a power-law behavior of the form $b(t) \sim t^{1 / 7}$ then the mode with the largest positive growth rate predicted by Paterson's analysis-which is used somewhat inconsistently because its derivation assumes that $d b / d t=0$-remains constant.

To assess to what extent Zheng et al.'s conclusions are affected by this inconsistency we note that for an arbitrary time-varying gap width $b(t)$, Eqs. (5) and (9) show that perturbations with wave number $k$ decay if

$$
\frac{d b}{d t}-\frac{(k-1)}{(k+1)} \frac{b}{t}+\frac{\mathcal{A}^{2} k(k-1)}{12 \sqrt{2} \mathrm{Ca}} \frac{b^{9 / 2}}{t^{3 / 2}}>0 .
$$




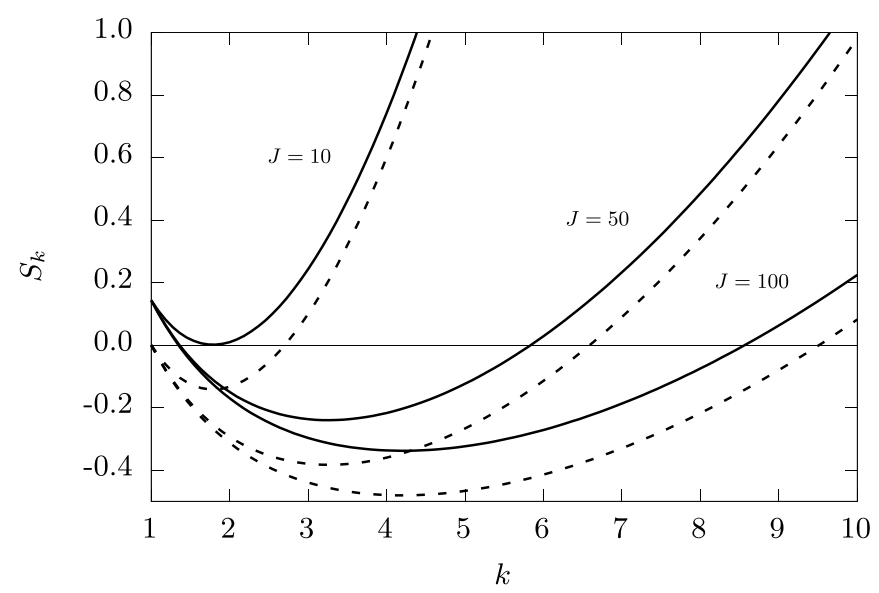

FIG. 2. Plot of the stability criterion Eq. (11) for $J=10,50$, and 100 . The axisymmetric state is unstable to nonaxisymmetric perturbations with integer wave number $k$ if $S_{k}<0$. The predictions corresponding to Zheng et al.'s approach [17] are shown by dashed lines.

For $b(t)=b_{1} t^{1 / 7}$ this condition becomes

$$
S_{k}=\frac{1}{7}-\frac{k-1}{k+1}+\frac{k(k-1)}{J}>0
$$

where

$$
J=\frac{12 \sqrt{2} \mathrm{Ca}}{\mathcal{A}^{2} b_{1}^{7 / 2}}=\frac{6 \mu Q^{3 / 2}}{\pi^{3 / 2} \gamma b_{1}^{* 7 / 2}}
$$

is precisely the control parameter appearing in Zheng et al.'s analysis. The inclusion of the $d b / d t$ term in our analysis results in the appearance of an additional term in the stability criterion Eq. (11) (the constant $1 / 7$ on the left-hand side of this equation), but its presence does not reintroduce a time-dependence into this condition. Thus, Zheng et al.'s key observation remains unchanged. Furthermore, the condition for a perturbation with wave number $k$ to have a negative instantaneous growth rate (implying linear stability with respect to such perturbations) can be expressed in terms of $J$ as

$$
J<J_{\mathrm{cr}}(k)=\frac{7 k\left(k^{2}-1\right)}{6 k-8},
$$

while, for a given, sufficiently large value of $J$, the wave number $k_{\max }$ of the perturbation with the largest positive growth rate satisfies

$$
J=\frac{7}{6}\left(3 k_{\max }^{2}-1\right)
$$

These results again only differ slightly from those obtained by Zheng et al.'s approach which yields $J_{\mathrm{cr}}(k)=k(k+1)$ and $J=3 k_{\max }^{2}-1$.

Figure 2 compares the two sets of results in a plot of the stability criterion Eq. (11) for three different values of $J$. Recall that the axisymmetric state is stable when $S_{k}>0$, therefore wave numbers for which $S_{k}<0$ represent unstable perturbations. Zheng et al.'s approach can be seen to consistently overestimate the growth-rate of the nonaxisymmetric perturbations because their analysis omits the stabilising effect of the $d b / d t$ term. However, both analyses show that the range of unstable wave numbers and the wave number with the fastest growth rate increase with $J$. 


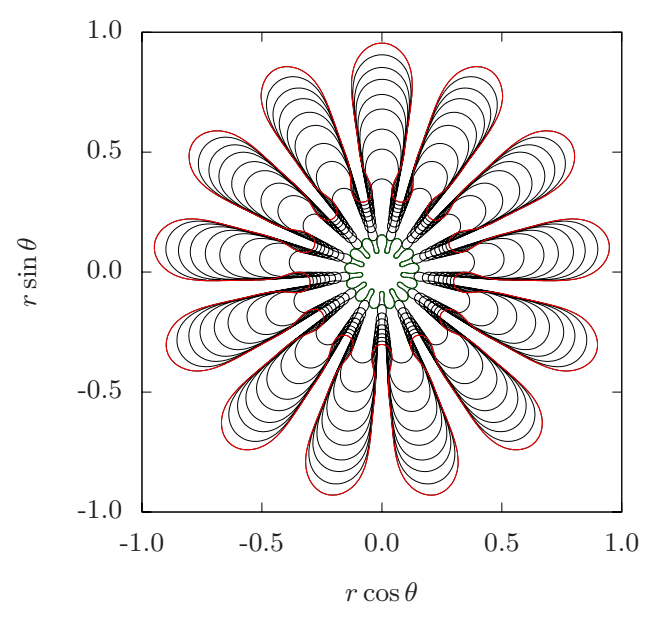

(a)

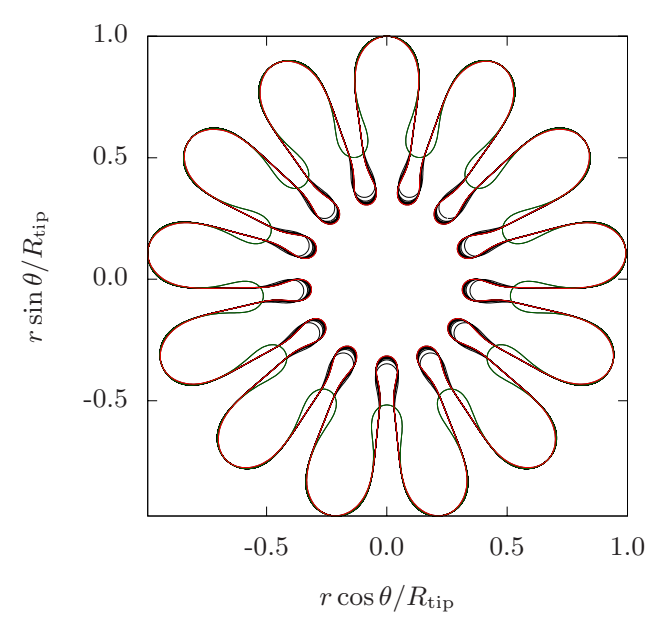

(b)

FIG. 3. (a) Series of snapshots showing the evolution of the interface for $J=600$ [28]. The simulation was started at $t_{0}=0.00125$ when the axisymmetric interface (of radius $R_{\text {init }}=0.05$ ) was subjected to an initial perturbation with wave number $k=k_{\max }=13$ and amplitude $\epsilon=5 \times 10^{-4}$. The innermost interface is shown for $t=0.011$ and the time interval between contours is $\Delta t=0.083$. (b) Interface contours from panel (a) scaled by the radius of the finger tip, $R_{\text {tip }}$. In panels (a) and (b), the first and the last interfaces in the sequence are highlighted with green and red colours, respectively.

\section{DIRECT NUMERICAL SIMULATIONS}

The results presented above confirm that for a lifting law of the form proposed by Zheng et al. [17], the wave number of the most rapidly growing small-amplitude perturbation remains constant throughout the system's evolution. To assess if this behavior is responsible for the development of the nonsplitting fingers observed in Zheng et al.'s experiments, we now conduct numerical simulations of the system's nonlinear evolution following the onset of the linear instability. For this purpose we used an oomph-lib-based [25] finite-element discretization of the governing equations, details of which can be found in Ref. [26].

All simulations were performed for $\mathrm{Ca}=0.3893$ and $R_{\text {init }}=0.05$ as in our previous work [26,27]. Suitable temporal and spatial convergence tests were performed to ascertain that the results presented below are fully converged. All simulations were stopped before the shape of the interface was significantly affected by the boundary of the finite cell at $r=1$.

\section{A. Unimodal perturbations and self-similar fingering}

We start by perturbing the initial circular bubble of radius $R_{\text {init }}=0.05$ with the single, most rapidly growing mode so that

$$
R\left(\theta, t=t_{0}\right)=R_{\text {init }}+\epsilon \cos \left(k_{\max } \theta\right),
$$

where we set the amplitude of the perturbation to $\epsilon=5 \times 10^{-4}$, i.e., $1 \%$ of the initial radius. Figure 3(a) shows the time-evolution of the interface for $J=600$ (for which $k_{\max }=13$ ), using snapshots of the interface plotted at regular time intervals. Thirteen identical finite-amplitude fingers grow from the initial perturbation and, interestingly, the fingers show no signs of splitting. Instead, they remain symmetric about their radial centreline and appear to approach a self-similar shape. This is confirmed in Fig. 3(b), where we rescaled the radial coordinate by the radius of the finger tip, $R_{\text {tip }}$, for each snapshot. This shows that, after an initial transient evolution that occurs within the first five contours shown in Fig. 3(b), the successive rescaled interfaces become virtually 


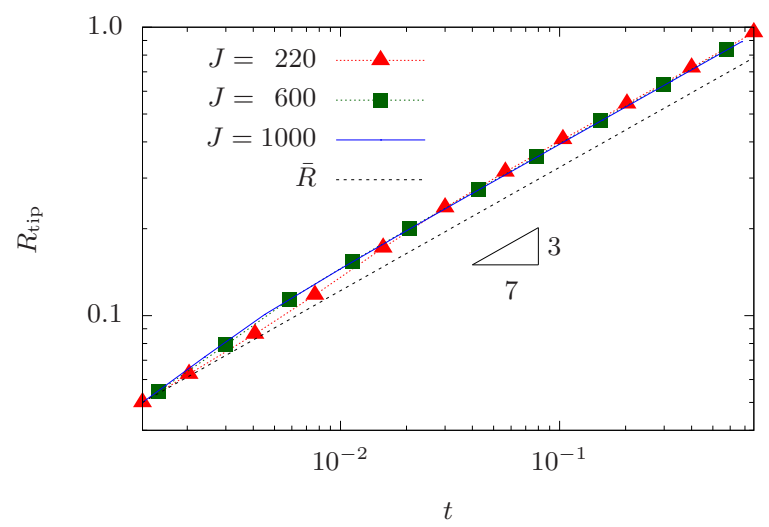

FIG. 4. Log-log plot of $R_{\text {tip }}$ as a function of time ${ }^{1}$ for $J=220,600$, and 1000 [30]. In each case, the interface is initially perturbed by the single, most-rapidly growing mode. The dotted line shows the radius of the axisymmetrically growing bubble, $\bar{R}(t)$.

indistinguishable. Equivalent behavior was observed in simulations for 14 different values of $J$ in the range $25 \leqslant J \leqslant 1000$, where in each case we imposed the most rapidly growing single-mode perturbation, with $k_{\max }$ given by Eq. (14).

In Fig. 4 we plot the tip radius, $R_{\text {tip }}$, as a function of time on a log-log scale for a range of $J$ values. As the tip radius increases from its initial value it rapidly approaches a power law behavior, $R_{\text {tip }} \sim t^{3 / 7}$, which is identical to that of the axisymmetrically growing bubble [see Eq. (5) and recall that $b(t) \sim t^{1 / 7}$ ]. This is again consistent with the observed self-similar evolution of the interface shape-if the bubble radius $R(t, \theta)$ approaches a self-similar behavior such that $R(t, \theta)=f(t) F_{k}(\theta)$, then volume conservation requires that $f(t) \sim t^{3 / 7}$. We note that the curves representing $R_{\text {tip }}(t)$ for different values of $J$ almost overlap, implying that the prefactor (which is always larger than that for the axisymmetrically growing bubble) is approximately independent of $J$, despite significant variations in $k_{\max }$, which ranges from 8 to 17 .

Next, we explore whether the interface shape evolves towards a self-similar solution when the initial single-mode perturbation has a wave number other than $k_{\max }$. For this purpose we choose a larger value of $J, J=1000$, to operate in a regime with a wide range of linearly unstable modes. We then perturb the initial circular bubble as in Eq. (15) but replace $k_{\max }$ with a wave number from the unstable range defined by Eq. (13). The resulting evolution of the interface is shown in Figs. 5(a) and 5(b) for different values of $k$, starting from a perturbation of amplitude $\epsilon=5 \times 10^{-4}$. In each case, the system undergoes a transient initial evolution but ultimately reaches a regime in which a number of identical, self-similar fingers have emerged from the initial perturbation. However, the number of self-similar fingers that develop depends on the wave number of the initial perturbation. For the perturbation with $k=k_{\max }=17$ [Fig. 5(a)] we retain the wave number of the initial perturbation. If we impose an initial perturbation with $k=7$ [Fig. 5(b)] the finite-amplitude fingers that develop from the initial perturbation split once (see the zoomed-in region shown in the inset) before approaching a self-similar pattern with 14 fingers. This implies that the number of self-similar fingers that can emerge from the initial perturbation is not directly related to the value of $k_{\max }$ from the linear stability analysis.

The tip splitting events that may occur during the early transients that precede the self-similar evolution of the interface are not restricted to tip doubling. This is illustrated in Fig. 5(c) where

\footnotetext{
${ }^{1}$ In a typical experiment, with $R_{\text {outer }}^{*}=20 \mathrm{~cm}, b_{0}^{*}=0.5 \mathrm{~mm}$ and $Q=25 \mathrm{ml} / \mathrm{min}$ as in Ref. [29], say, the total simulation time corresponds to approximately $150 \mathrm{~s}$ of interface expansion before the outer boundary of the cell is reached.
} 
(a)

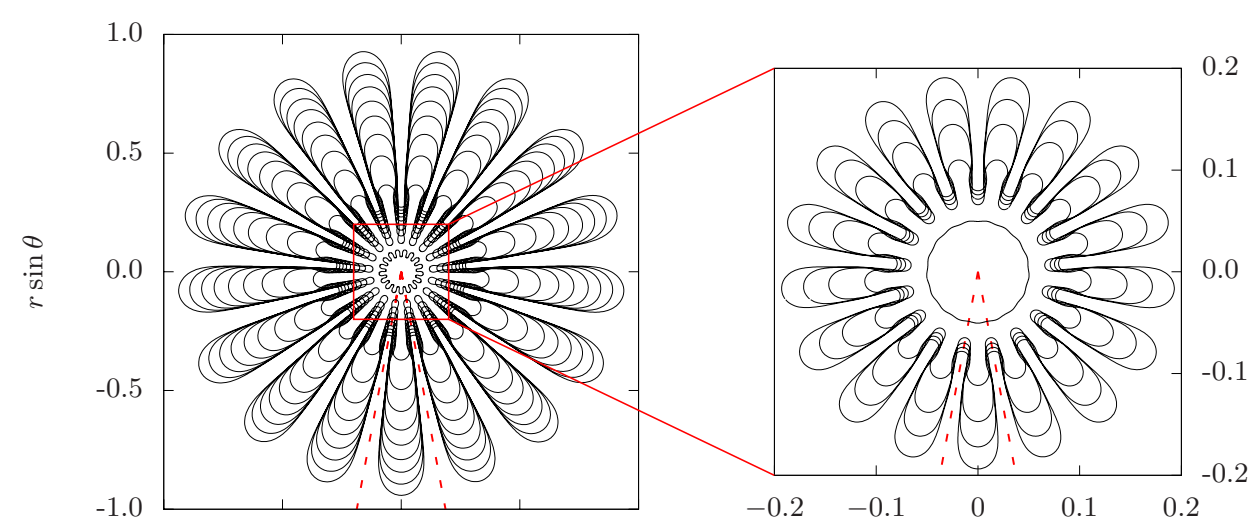

(b)

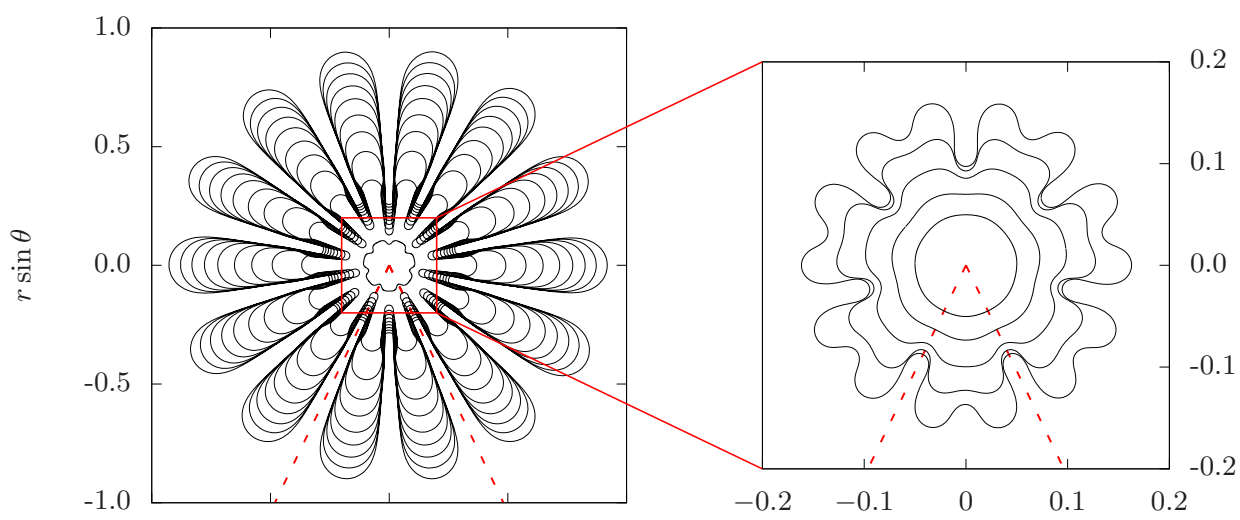

(c)

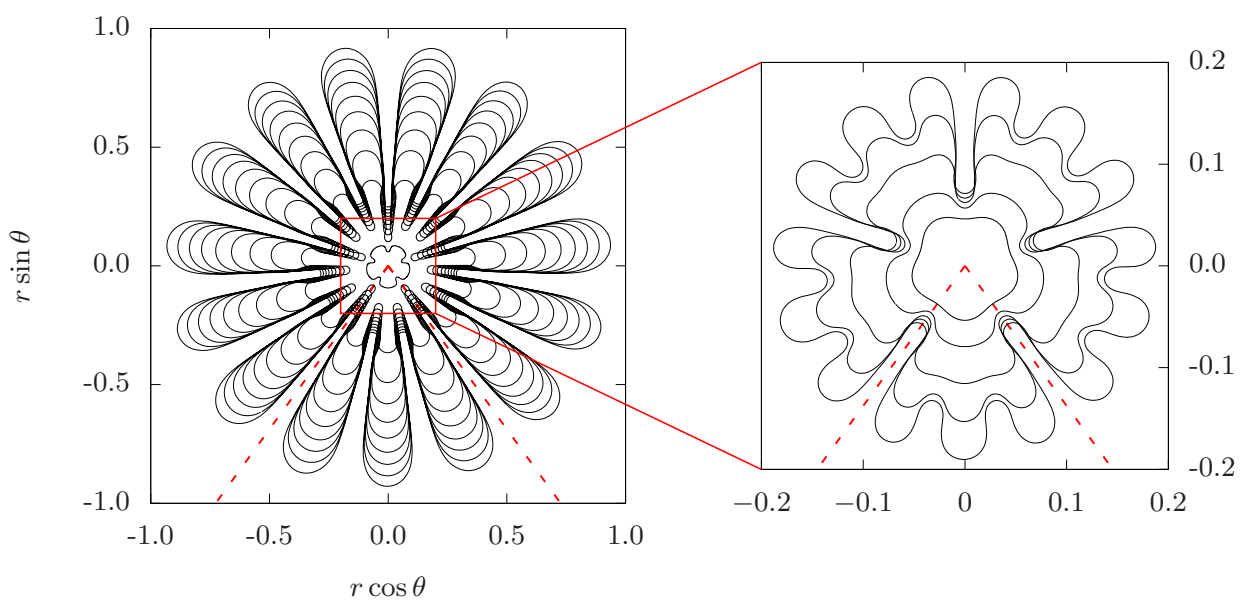

FIG. 5. Series of snapshots showing the evolution of the interface for $J=1000$ [28]. The simulations were started at $t_{0}=0.00125$ when the axisymmetric interface (of radius $R_{\text {init }}=0.05$ ) was subjected to an initial perturbation with wave number (a) $k=k_{\max }=17$, (b) $k=7$, (c) $k=5$. The amplitude of the initial perturbation is $\epsilon=5 \times 10^{-4}$ for panels (a) and (b) and $1.25 \times 10^{-2}$ for panel (c). The time interval between contours is (a) $\Delta t=0.069$, (b) $\Delta t=0.078$, (c) $\Delta t=0.08$. In each case the first contour is shown at $t=0.011$. Dashed lines indicate a sector within which a single finger develops at early times. The insets show the early evolution of the interface with successive contours chosen so that the average radius $\langle R\rangle=\frac{1}{2 \pi} \int_{0}^{2 \pi} R d \theta$ increases by 0.022 . 


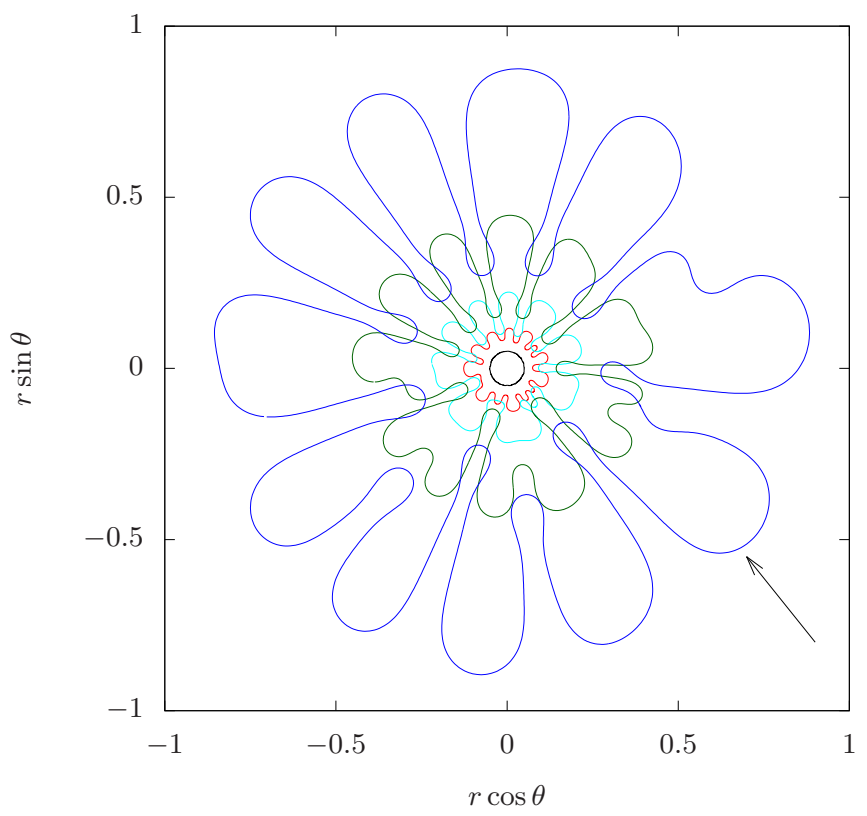

FIG. 6. Instantaneous interface shapes with average radius $\langle R\rangle=0.05$ (black), 0.1 (red), 0.17 (cyan), 0.31 (dark green), and 0.6 (blue), respectively, computed for $J=600$ [28]. The arrow points to a region of the interface that undergoes tip-splitting and finger competition.

we imposed an initial perturbation with $k=5$ and also increased the amplitude of the perturbation to $25 \%$ of the initial radius. The early time growth of the initial perturbation is now followed by a tip-tripling event (see the zoomed-in region shown in the inset) before the bubble approaches a configuration with 15 self-similar fingers.

For all cases shown in Fig. 5 the imposed unimodal initial perturbation is symmetric about the radial centreline of a sector spanned by an angle $2 \pi / k$, shown by the dashed lines. Even when the evolution of the interface involved tip-splitting into two or three fingers, the newly formed fingers did not compete with each other to break this initial symmetry. The fact that the computations were performed with unstructured meshes which (despite being sufficiently fine to ensure meshindependent solutions) inevitably introduce small nonaxisymmetric perturbations, suggests that the self-similar fingers are linearly stable.

\section{B. Nonunimodal perturbations and disordered fingering}

Unimodal initial perturbations, such as those imposed in the simulations presented in the previous section, are, of course, difficult to realize in actual experiments. The initial perturbation to a real bubble is likely to comprise a wide range of modes. To analyze this scenario we now perturb the interface with the full range of linearly unstable wave numbers. We introduce these perturbations by adding all of the unstable modes to the initial shape of the interface, each with a randomly generated (and uniformly distributed) relative amplitude $\hat{R}_{k} \in[0,1]$ and phase $\phi_{k} \in[0,2 \pi$ ),

$$
R\left(\theta, t=t_{0}\right)=R_{\text {init }}+\epsilon \sum_{\text {unstable } k} \hat{R}_{k} \cos \left(k \theta+\phi_{k}\right),
$$

where the overall amplitude $\epsilon$ was chosen to be $0.5 \%$ of the initial radius. We performed 50 simulations for 14 values of $J$ within the range $25 \leqslant J \leqslant 1000$. 
A typical evolving interface is shown in Fig. 6 for $J=600$ for which $k_{\max }=13$. The snapshots of the interface are shown for different values of the average radius $\langle R\rangle$ and are plotted in different colours. When the mean radius has grown to $\langle R\rangle=0.1$ (red) the random initial perturbation has led to the development of 13 distinct fingers of varying widths and lengths. Differences in the length of adjacent fingers are amplified as the fingers grow (finger competition) and when $\langle R\rangle=0.17$ (cyan), the total number of fingers has decreased from 13 to 10 . At $\langle R\rangle=0.17$ (cyan) the four largest fingers have broadened considerably so that their tips are approximately flat. When $\langle R\rangle=0.31$ (dark green) these fingers have undergone a tip-doubling followed by competition between the newly formed fingers, while another finger has broadened sufficiently for another tip-doubling to occur. Because the fingers are not symmetric about their radial centreline, the tip-doubling events lead to pairs of fingers of different shapes and radial tip positions, so that one of the fingers screens the other and adopts a strongly asymmetric shape; see, for example, the finger identified by the arrow in Fig. 6 . The final pattern shown in Fig. 6 (blue) at $\langle R\rangle=0.6$ comprises 11 distinct fingers of different shapes, which continue to evolve and interact as they grow.

We characterize the patterns in Fig. 6 by following Ref. [17] and compute the Fourier coefficients $c(k)$ of $R-\langle R\rangle$,

$$
c(k)=\frac{1}{2 \pi} \int_{-\pi}^{\pi}(R-\langle R\rangle) \exp (-i k \theta) d \theta .
$$

To compare coefficients computed for different values of $\langle R\rangle$, we normalise the amplitudes $c(k)$ by the area under the corresponding spectral curves and denote the normalized amplitudes by $\|c(k)\|$. This allows us to focus on the relative amplitudes of the modes rather than their absolute value which grows with increasing radius.

Figure 7 shows that the evolution of the spectral curves is closely correlated with the evolution of the interfacial pattern. The interface shape can be seen to comprise a mixture of modes, with the number of distinct fingers observed at the interface approximately corresponding to the mode with the maximum normalized amplitude, which we label $K_{\max }$.

In all the simulations performed with random initial perturbations the value of $K_{\max }$ continued to fluctuate throughout the system's evolution. Typically, $K_{\max }$ decreased significantly from its initial value at early times when many modes had comparable amplitudes [see Fig. 7(a)]. At later times, $K_{\max }$ was more likely to increase due to tip-splitting events. The overall rate of change of $K_{\max }$ decreased with increasing time, but $K_{\max }$ continued to fluctuate until fingers reached the edge of the computational domain. This scenario was observed for all the values of $J$ investigated $(J \in[25,1000])$, despite the fact that the growth rate of the imposed small-amplitude initial perturbation is smaller for smaller values of $J$.

Figure 8 shows how variations in $J$ affect $K_{\max }$ which we evaluated when $\langle R\rangle=0.6$ - the largest value reached in all simulations (which necessarily terminate when one of the fingers reaches the outer edge of the computational domain). The error bars on the symbols quantify the standard deviation of $K_{\max }$ across 50 runs for each value of $J$ and highlight the disordered nature of the interface evolution. The two lines show the most unstable wave number $k_{\max }$ predicted by our linear stability analysis (solid) and by Zheng et al.'s approach (dashed). For modest values of $J$ the wave number $K_{\max }$ observed in the numerical simulations is remarkably close to $k_{\max }$ from the linear stability analysis. However, for larger values of $J, K_{\max }$ remains significantly below $k_{\max }$. Furthermore, none of the simulations that were started with random initial perturbations showed any signs of settling on a self-similar state-the fingers continued to split and then compete with each other over the entire range of the simulations.

Our results show that the system's behavior is fundamentally different when the initial, axisymmetrically expanding bubble is subjected to unimodal or nonunimodal perturbations to its shape. This raises the question in what way the small-amplitude nonunimodal perturbations that we deliberately introduced in the computations shown in Fig. 6 differ from those introduced unintentionally by the use of unstructured meshes in the simulations in Fig. 5, say. Clearly, the deliberately introduced unimodal perturbation imposed by Eq. (15) must have a larger initial 


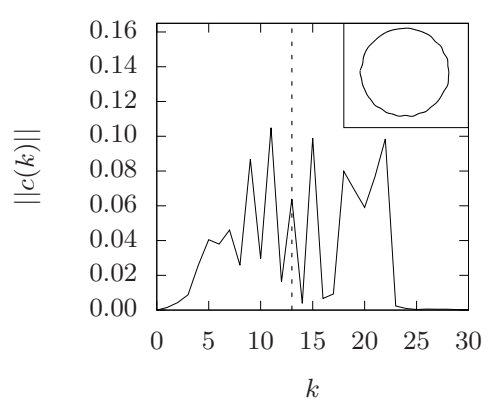

(a)

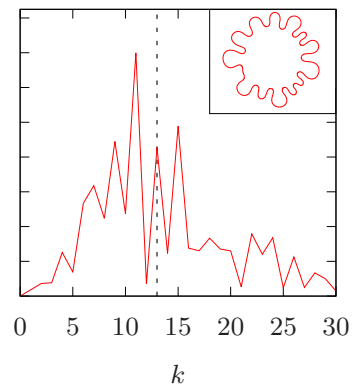

(b)

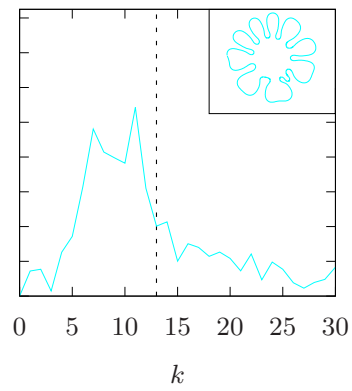

(c)

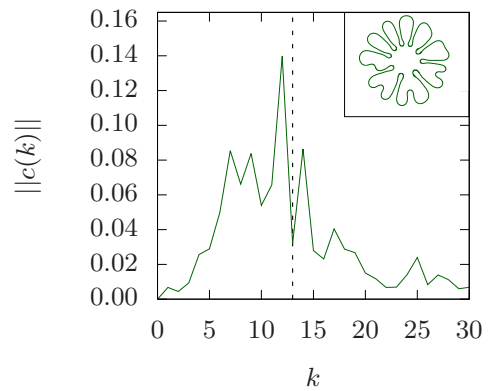

(d)

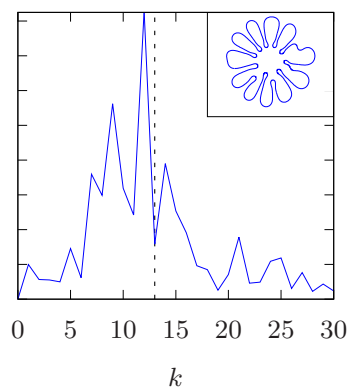

(e)

FIG. 7. Normalized spectra of the instantaneous interface shapes shown in Fig. 6 for $J=600$. The mean radii are (a) $\langle R\rangle=0.05$, (b) 0.1 , (c) 0.17 , (d) 0.31 , and (e) 0.6 [30]. The vertical dashed line indicates the value of $k_{\max }=13$ predicted by the linear stability analysis.

amplitude than those caused by the unstructured mesh. This was ensured by performing the computations on sufficiently fine meshes. However, we also have to make sure that the growth rate of the deliberately introduced unimodal perturbation is sufficiently large so that it deforms the interface into a nonlinear regime before the other modes have grown to comparable amplitude.

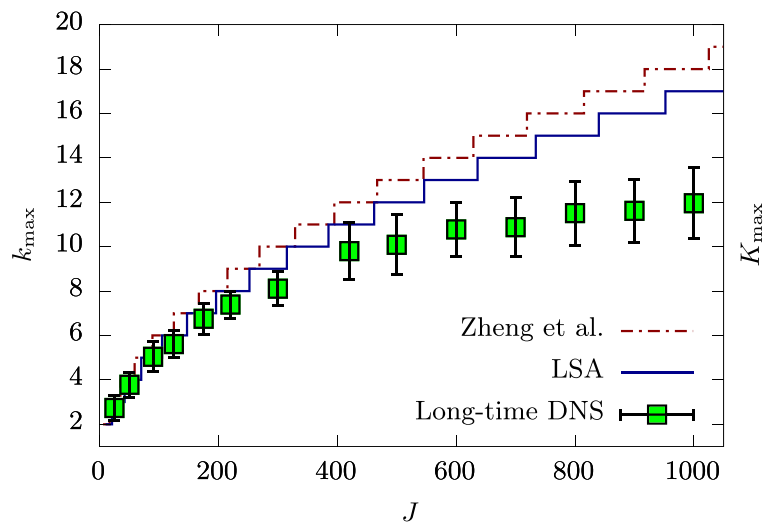

FIG. 8. The variation of $K_{\max }$ with $J$ from direct numerical simulations (DNS) [30]. The error bars denote the standard deviation across 50 simulations. The lines show $k_{\max }$ from the results of the linear stability analysis, using our own predictions (solid) and those from Ref. [17] (dashed). $k_{\max }$ can only take integer values, which we connect by vertical line segments, resulting in steps in the curves. 
This is, of course, easiest to achieve if the deliberately introduced perturbation is the one with the maximum growth rate, as in Fig. 5(a) where we applied a perturbation with $k=k_{\max }=17$. The initial amplitude of $\epsilon=5 \times 10^{-4}$ used for the perturbation with $k=7<k_{\max }$ in Fig. 5(b) sufficed to create seven finite-amplitude fingers that subsequently underwent a single tip-splitting event before approaching the self-similar regime. However, when using that amplitude to impose a perturbation with $k=5$ (which has a much smaller but still positive growth rate) the system evolved in a disordered manner, with unequal fingers that kept splitting and competing with each other. The fivefold overall symmetry of the initial perturbation could only be retained by increasing its amplitude, as in Fig. 5(c), where the initial fingers undergo a tip-tripling before approaching a self-similar regime with 15 nonsplitting fingers.

\section{SUMMARY AND DISCUSSION}

We studied the dynamics of an expanding gas bubble that displaces a viscous liquid in a radial Hele-Shaw cell with a time-varying gap width, focusing on the case of a $t^{1 / 7}$ power-law for the plate separation. Our full linear stability analysis confirmed Zheng et al.'s [17] prediction that the wave number $k_{\max }$ of the most unstable small-amplitude perturbation to the axisymmetrically expanding bubble is independent of the bubble's radius. We then employed numerical simulations to follow the growth of the instability into the finite-amplitude regime. This showed that for unimodal perturbations with a wave number that matches the most unstable wave number from the linear stability analysis, self-similar fingers formed on the interface. This is in stark contrast to the typical behavior observed in Hele-Shaw cells with constant gap widths where fingers tend to split and compete with each other, resulting in complex, disordered, and continuously evolving interface shapes.

Interestingly, self-similar fingers also emerged from unimodal perturbations with wave numbers other than $k_{\max }$. In this case, the finite-amplitude fingers tended to pass through an initial transient period during which they split (via tip doubling or tripling) before ultimately approaching a final regime in which their shapes evolved in a self-similar fashion without any further splitting. The number of identical finite-amplitude, self-similar fingers emerging from this process depended on the initial condition. The self-similar fingers appear to be linearly stable in the sense that they persist despite inevitable small perturbations due to the use of unstructured meshes in the simulations. The fact that the self-similar solutions can only be realized from unimodal perturbations with wave number $k$, so that the interface was symmetric about the radial centreline of a sector spanned by an angle $2 \pi / k$, suggests that they are unlikely to be observable in actual experiments (unless an experiment happens to be performed in the narrow regime where there is only a single unstable mode).

When starting the simulations from nonunimodal, random perturbations to the initial bubble shape-a scenario more representative of the situation in actual physical experiments-multiple, competing, and continuously splitting fingers developed, similar to the behavior typically found in the constant-gap system. For modest values of the control parameter $J$, the wave number $K_{\max }$ of the dominant mode contained in the Fourier spectrum of the finite-amplitude fingers tended to be close to $k_{\max }$ (as observed in Zheng et al.'s experiments) but for larger values of $J$ we found $K_{\max }$ to be significantly smaller than $k_{\max }$, suggesting that there is no straightforward relationship between the two quantities. This is in contrast to the observations of Li et al. [24], who controlled the injection flux in a constant-gap Hele-Shaw cell according to the power law $Q_{1} t^{*-1 / 3}$. Their linear stability analysis showed that, as in our system, the number of unstable modes and the most unstable wave number remained constant as the interface expanded. However, Li et al. found stable selfsimilar patterns emerging from random initial perturbations, with the number of finite-amplitude self-similar fingers exactly equal to the most unstable wave number from the linear stability analysis. While the observed correlation between the most unstable wave number from the linear stability analysis and the number of finite-amplitude self-similar fingers is intriguing, it is important to stress that it is unclear how the fact that the wave number of the most-unstable small-amplitude 
perturbation to the axisymmetric bubble remains independent of its radius could affect the behavior of the finite-amplitude fingers that emerge from the linear instability: tip-splitting of finite-amplitude fingers occurs in the course of their nonlinear evolution (and does not necessarily involve the occurrence of a secondary instability), rather than because the most unstable wave number of perturbations to some (far away) axisymmetric state changes. We therefore suggest to interpret the nonsplitting fingers observed in our system as stable self-similar solutions of the governing equations. Equally, the nonsplitting fingers found by Li et al. [24] are stable self-similar solutions of their governing equations. They presumably have a different basin of attraction compared to the self-similar solutions found in our study given the different outcomes of the long-time simulations with random initial perturbations. Nevertheless, the self-similar fingers found in both problems appear to be analogues to the steadily propagating fingers that develop in rectangular Hele-Shaw channels $[1,22,23,31]$. We also observed the systematic transient evolution of the interface towards these self-similar states via tip-doubling and tripling. By analogy with rectangular Hele-Shaw channels, this suggests that in radial Hele-Shaw cells other unstable self-similar solutions may exist which are reminiscent of the unstable Romero-Vanden-Broeck multiple-tip solutions [31].

\section{ACKNOWLEDGMENTS}

The authors thank Andrew Hazel, Scott McCue, and Liam Morrow for helpful discussions. The work of A.J. was funded through EPSRC Grant No. EP/P026044/1. The research data supporting this publication can be found in the Supplemental Material $[28,30]$.

[1] P. G. Saffman and G. I. Taylor, The penetration of a fluid into a porous medium or Hele-Shaw cell containing a more viscous liquid, Proc. Roy. Soc. London A 245, 312 (1958).

[2] L. Paterson, Radial fingering in a Hele-Shaw cell, J. Fluid Mech. 113, 513 (1981).

[3] J. D. Chen, Growth of radial viscous fingers in a Hele-Shaw cell, J. Fluid Mech. 201, 223 (1989).

[4] Y. Couder, Viscous fingering as an archetype for growth patterns, in Perspectives in Fluid Mechanics-A Collective Introduction to Current Research, edited by G. K. Batchelor, H. K. Moffat, and M. G. Worster (Cambridge University Press, Cambridge, 2000), pp. 53-104.

[5] E. Sharon, M. G. Moore, W. D. McCormick, and H. L. Swinney, Coarsening of Fractal Viscous Fingering Patterns, Phys. Rev. Lett. 91, 205504 (2003).

[6] E. Ben Jacob, H. Schmueli, O. Shochet, and A. Tenenbaum, Adaptive self-organisation during growth of bacterial colonies, Physica A 187, 378 (1992).

[7] W. W. Mullins and R. F. Sekerka, Stability of a planar interface during solidification of a dilute binary alloy, J. Appl. Phys. 35, 444 (1964).

[8] R. S. Seright, Cleanup of oil zones after a gel treatment, SPE Product. Operat. 21, 237 (2006).

[9] A. A. Osiptsov, Fluid mechanics of hydraulic fracturing: A review, J. Petrol. Sci. Eng. 156, 513 (2017).

[10] T. T. Al-Housseiny, P. A. Tsai, and H. A. Stone, Control of interfacial instabilities using flow geometry, Nat. Phys 8, 747 (2012).

[11] G. Bongrand and P. A. Tsai, Manipulation of viscous fingering in a radially tapered cell geometry, Phys. Rev. E 97, 061101(R) (2018).

[12] D. Pihler-Puzović, P. Illien, M. Heil, and A. Juel, Suppression of Complex Fingerlike Patterns at the Interface Between Air and a Viscous Fluid by Elastic Membranes, Phys. Rev. Lett. 108, 074502 (2012).

[13] D. Pihler-Puzović, G. G. Peng, J. R. Lister, M. Heil, and A. Juel, Viscous fingering in a radial elasticwalled Hele-Shaw cell, J. Fluid Mech. 849, 163 (2018).

[14] A. Juel, D. Pihler-Puzović, and M. Heil, Instabilities in blistering, Ann. Rev. Fluid Mech. 50, 691 (2018).

[15] E. O. Dias, E. Alvarez-Lacalle, M. S. Carvalho, and J. A. Miranda, Minimization of Viscous Fluid Fingering: A Variational Scheme for Optimal Flow Rates, Phys. Rev. Lett. 109, 144502 (2012). 
[16] I. Bischofberger, R. Ramachandran, and S. R. Nagel, Fingering versus stability in the limit of zero interfacial tension, Nat. Commun. 5, 5265 (2014).

[17] Z. Zheng, H. Kim, and H. A. Stone, Controlling Viscous Fingering Using Time-Dependent Strategies, Phys. Rev. Lett. 115, 174501 (2015).

[18] T. Sadhu and D. Dhar, Modelling proportionate growth, Curr. Sci. 103, 512 (2012).

[19] T. Thomé, M. Rabaud, V. Hakim, and Y. Couder, The Saffman-Taylor instability: From the linear to the circular geometry, Phys. Fluids A 1, 224 (1989).

[20] E. Lajeunesse and Y. Couder, On tip splitting instability of viscous fingers, J. Fluid Mech. 419, 125 (2000).

[21] R. Combescot and M. Ben Amar, Selection of Saffman-Taylor Fingers in the Sector Geometry, Phys. Rev. Lett. 67, 453 (1991).

[22] M. Ben Amar, Exact self-similar shapes in viscous fingering, Phys. Rev. A 43, 5724 (1991).

[23] M. Ben Amar, V. Hakim, M. Mashaal, and Y. Couder, Self dilating fingers in wedge-shaped Hele-Shaw cell, Phys. Fluids A 3, 1687 (1991).

[24] S. Li, J. S. Lowengrub, J. Fontana, and P. Palffy-Muhoray, Control of Viscous Fingering Patterns in a Radial Hele-Shaw Cell, Phys. Rev. Lett. 102, 174501 (2009).

[25] M. Heil and A. L. Hazel, oomph-lib-An object-oriented multi-physics finite-element library, in Fluid-Structure Interaction, edited by M. Schäfer and H.-J. Bungartz (Springer, Berlin, 2006), pp. 19-49; oomph-lib is available as open-source software at http://www.oomph-lib.org.

[26] D. Pihler-Puzović, R. Périllat, M. Russell, A. Juel, and M. Heil, Modeling the suppression of viscous fingering in elastic-walled Hele-Shaw cells, J. Fluid Mech. 731, 162 (2013).

[27] D. Pihler-Puzović, A. Juel, and M. Heil, The interaction between viscous fingering and wrinkling in elastic-walled Hele-Shaw cells, Phys. Fluids 26, 022102 (2014).

[28] See Supplemental Material at http://link.aps.org/supplemental/10.1103/PhysRevFluids.4.064002 for videos of expanding interface generated using the same data.

[29] D. Pihler-Puzović, A. Juel, G. G. Peng, J. R. Lister, and M. Heil, Displacement flows under elastic membranes. Part 1: Experiments and direct numerical simulations, J. Fluid Mech. 784, 487 (2015).

[30] See Supplemental Material at http://link.aps.org/supplemental/10.1103/PhysRevFluids.4.064002 for the numerical data used to generate this figure.

[31] A. Franco-Gómez, A. B. Thompson, A. L. Hazel, and A. Juel, Sensitivity of Saffman-Taylor fingers to channel-depth perturbations, J. Fluid Mech. 794, 343 (2016). 